\title{
Descemet's scroll in syphilitic interstitial keratitis: a case report with anterior segment evaluation and a literature review
}

This article was published in the following Dove Press journal:

International Medical Case Reports Journal

28 September 2015

Number of times this article has been viewed

\author{
Ngamjit Kasetsuwan ${ }^{1,2}$ \\ Usanee Reinprayoon ${ }^{1,2}$ \\ Patchima Chantaren ${ }^{2}$ \\ 'Department of Ophthalmology, \\ Faculty of Medicine, Chulalongkorn \\ University, ${ }^{2} \mathrm{Ophthalmology}$ \\ Department, King Chulalongkorn \\ Memorial Hospital, Bangkok, Thailand
}

Purpose: To present a case of syphilitic interstitial keratitis with Descemet's scrolls, as well as its characteristic findings in an anterior segment investigation in relation to the histopathologic findings from a literature review.

Case presentation: A case report of a 64-year-old woman with syphilitic infection presented with band keratopathy and retrocorneal scrolls. Slit-lamp photography, confocal microscopy, anterior segment optical coherence tomography (AS-OCT), and ultrasound biomicroscopy were performed. Four previous reports were reviewed to describe the pathogenesis, natural history, and histopathologic and immunohistologic findings of the Descemet's scroll.

Results: The spectacle-corrected visual acuity was 20/50 and 20/30 in the right and left eyes, respectively. The scrolls appeared as a translucent web extending from Descemet's membrane into the anterior chamber. Confocal microscopy showed decreased endothelial cell density, pleomorphism, polymegathism, and hyperreflective fibrocellular rods with central hollow. The AS-OCT and ultrasound biomicroscopy showed rod-shaped retrocorneal scrolls. The corneal thickness was 494 microns, as measured by AS-OCT. The corneal lesion remained stable during the 6-year follow-up period without the need for keratoplasty, while the previous literature reported spontaneous and postoperative corneal decompensation.

Conclusion: We present a case of syphilitic interstitial keratitis with rare Descemet's scrolls featuring relatively good visual acuity. Although we did not obtain the corneal tissue for examination, the anterior segment investigation provides insight into the underlying histopathology and natural disease history. The central hollow and cellular component seen during confocal microscopy might correspond to the amorphous core and the abnormal endothelial cells in the histopathologic findings. Failure to detect the corneal endothelium due to stromal opacity in these cases is possible, however, compromised endothelium may present.

Keywords: confocal microscopy, Descemet's membrane, endothelium, retrocorneal membrane, retrocorneal scrolls

\section{Introduction}

Interstitial keratitis is a nonsuppurative inflammation of the corneal stroma without primary involvement of the epithelium or endothelium. Syphilitic interstitial keratitis was the most common interstitial keratitis in the preantibiotic era. Most cases have been associated with congenital syphilis. The clinical manifestations of interstitial keratitis include tearing, photophobia, perilimbal injection, corneal stromal inflammation, keratic precipitates, stromal neovascularization, corneal edema, and corneal opacity or scarring in late stages of the disease. ${ }^{1}$ Retrocorneal scrolls or scrolling of Descemet's membrane is a rare finding reported in syphilitic interstitial keratitis. Our literature search identified two cases reported in 1983 and 2001, 2,3 and two case series
Correspondence: Ngamjit Kasetsuwa Department of Ophthalmology, Faculty of Medicine, Chulalongkorn University, I873, Rama 4 Road,

Pathumwan, Bangkok 10330, Thailand

Tel +6622564l42

Email ngamjitk@gmail.com
International Medical Case Reports Journal 2015:8 219-223 (c) (i) (-) 2015 Kasetsuwan et al. This work is published by Dove Medical Press Limited, and licensed under Creative Commons Attribution - Non Commercial (unported, v3.0)

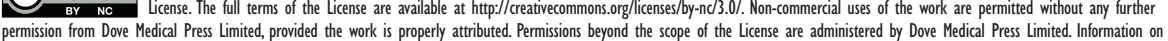
permission from Dove Medical Press Limited, provided the work is properly attributed. P 
of 14 eyes and five eyes with syphilitic retrocorneal scrolls in $1976^{4}$ and $2007,{ }^{5}$ respectively. The patients in the reported cases underwent penetrating keratoplasty due to pseudophakic bullous keratopathy or corneal opacity and edema. We present a patient with syphilitic interstitial keratitis and retrocorneal scrolls who had relatively good visual acuity (VA) and a characteristic finding observed during an anterior segment evaluation that had never been reported.

\section{Case presentation}

A 64-year-old woman presented to King Chulalongkorn Memorial Hospital, Bangkok, Thailand, in 2007 with irritation in the right eye from band keratopathy. The patient gave informed consent to this case presentation. At the time of this study, the Institutional Review Board did not require approvals for this type of article. She reported a remote history of bilateral red eyes with decreased vision at the age of 15 years in 1964 that improved after the injection of kanamycin. The Treponema pallidum haemagglutination assay test was positive, while the venereal disease research laboratory and human immunodeficiency virus antibody tests were negative. Her neurologic examination performed by an internist was unremarkable. Her best spectacle-corrected VA upon her first visit was 20/50 in the right eye and 20/30 in the left eye. The intraocular pressure values were $10 \mathrm{mmHg}$ and $9 \mathrm{mmHg}$ in the right and left eyes, respectively. There was no substantial stromal opacity or corneal edema. The Descemet's scrolls appeared as multiple translucent rods with a central gray core, and they were arranged in a web and extended into the anterior chamber (Figure 1). Few guttae were observed. The involvement in the left eye was milder, with fine retrocorneal scrolls but dense stromal ghost vessels (Figure 2). A pupillary membrane was also present in the right eye and Koeppe nodules were evident in the left eye (Figure 3). At the 6-year follow-up examination, the corneal lesion remained stable, but the patient's vision had deteriorated slightly due to cataract formation, with best spectacle-corrected VA values of $20 / 70$ in the right eye and 20/50 in left eye, respectively. The anterior segment evaluation was performed at the last visit.

\section{Anterior segment evaluation Confocal microscopy}

Confocal microscopy, using a ConfoScan4 (Nidek Inc., Fremont, CA, USA) showed endothelial pleomorphism and polymegathism. The endothelial cell density values were 587 cells $/ \mathrm{mm}^{2}$ and 1,082 cells $/ \mathrm{mm}^{2}$ in the right and left eyes, respectively. No guttae were observed (Figure 4). The scrolls appeared as tubular structures, with outer hyperreflectivity and inner hyporeflectivity. Some areas had cellular components on the surface (Figure 5). The epithelium and keratocytes appeared normal.

\section{Anterior segment optical coherence tomography}

Anterior segment optical coherence tomography (AS-OCT) (Spectralis ${ }^{\circledR}$ HRA, Heidelberg Engineering GmbH, Heidelberg,
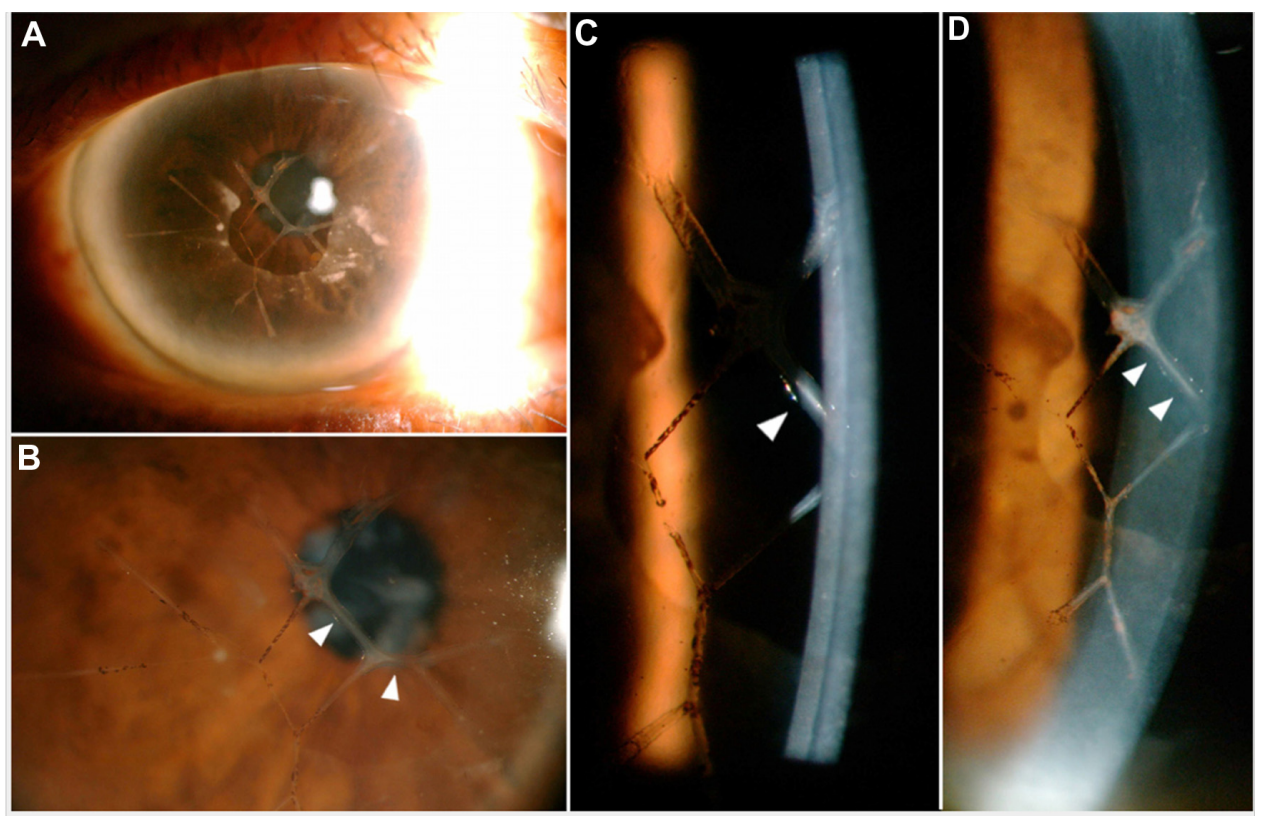

Figure I Slit-lamp photograph of the right eye.

Notes: (A) Slit-lamp photograph of the right eye. (B) Descemet's scroll, indicated by the arrowheads. (C) The Descemet's scroll appears as a translucent rod with a central core and (D) it extends into the anterior chamber. 

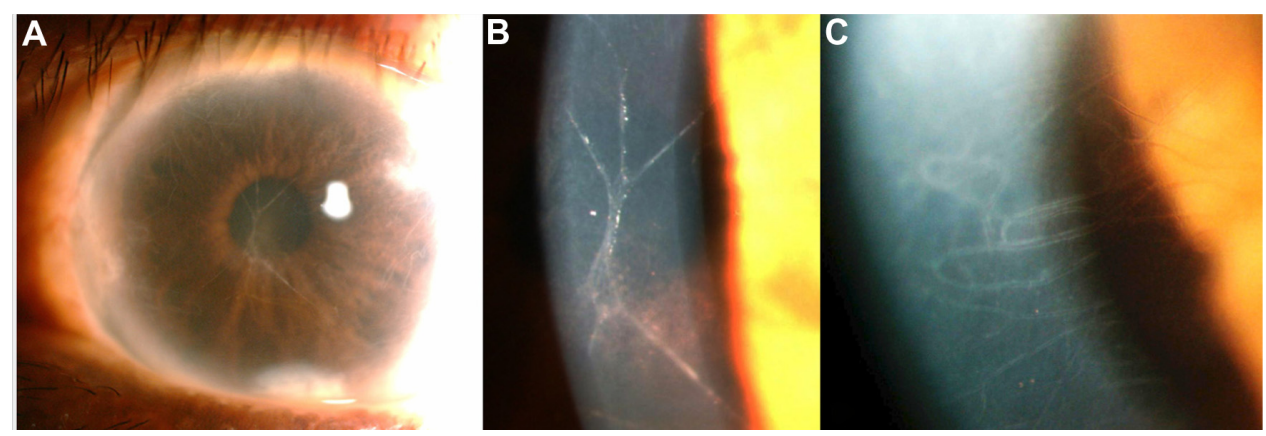

Figure 2 Slit-lamp photograph of the left eye.

Note: (A) Slit-lamp photograph of the left eye (B) showing a fine Descemet's scroll and (C) stromal ghost vessels.

Germany) showed a thickened Descemet's membrane. The scrolls extended from the Descemet's membrane into the anterior chamber. The outer portion of the scrolls appeared to be hyperreflective when compared with the inner portion, which was hyporeflective. There was no stromal opacity and the central cornea was 494 microns thick (Figure 6A and B).

\section{Ultrasound biomicroscopy}

The ultrasound biomicroscopy (UBM) $50 \mathrm{~Hz}$ linear scanner (QM Aviso; Quantel Medical, Pérignat-lès-Sarliève, France) displayed centrally hollow rods with a hyperechoic wall (Figure 6C and D).

\section{Discussion}

Retrocorneal scrolls, or scrolling of Descemet's membrane, are a rare finding in syphilitic interstitial keratitis. They occur in the late stage of the disease, ${ }^{3}$ are commonly seen in conjunction with congenital syphilis, and often first appear many years after birth. ${ }^{5}$ The retrocorneal scrolls can also be found in association with other types of interstitial keratitis, previous perforating injury, congenital glaucoma, ${ }^{6}$ and tears in Descemet's membrane associated with birth trauma. ${ }^{7}$
In chronic interstitial keratitis, the regenerating corneal endothelium produces a spectrum of changes in Descemet's membrane. Waring et $\mathrm{al}^{4}$ proposed that the mechanisms of Descemet's scroll formation included an inflammatory insult to a relatively young endothelium, changes in the endothelial function to fibroblast-like activity, the production of abnormal basement membrane material, and the formation of a new multilaminar Descemet's membrane.

In 1983, Scattergood et $\mathrm{al}^{2}$ reported the clinicopathologic features of a 45 -year-old woman with syphilitic interstitial keratitis and extensive retrocorneal scrolls who developed bullous keratopathy after uneventful cataract extraction. In 2001, Kawaguchi et $\mathrm{al}^{3}$ also described the histology and immunohistochemistry of a corneal button from a 56-year-old woman with syphilitic interstitial keratitis and retrocorneal scrolls, who underwent penetrating keratoplasty 4 years after extracapsular cataract extraction. Specular microscopy failed to detect corneal endothelium due to stromal opacity at the time of cataract surgery. ${ }^{3}$ In 2007, Dogru et $\mathrm{al}^{5}$ published the most recent report of a case series. All five eyes in that study had a best-corrected distance VA of below 20/200 due to corneal opacity with retrocorneal

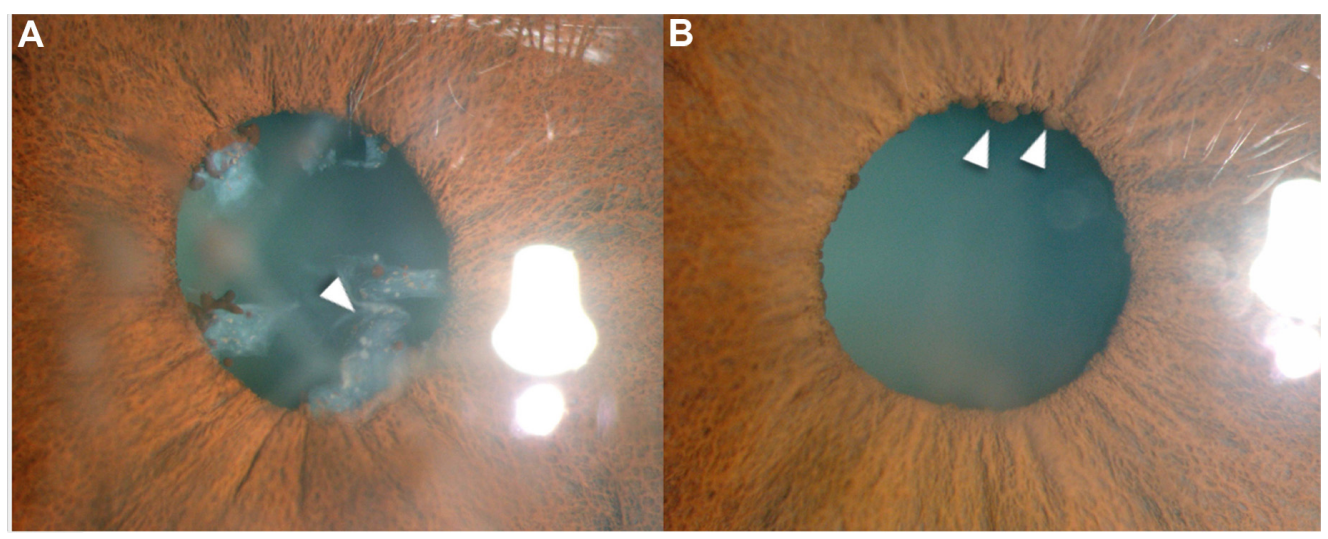

Figure 3 Slit-lamp photograph.

Note: (A) Arrowhead shows pupillary membrane in the right eye and (B) Arrowheads show Koeppe nodules in the left eye. 


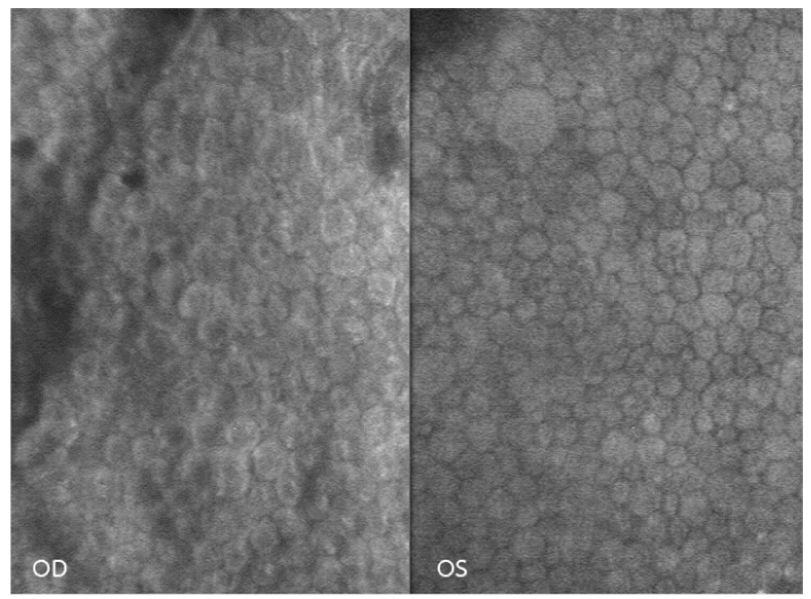

Figure 4 OD and OS confocal microscopy show endothelial pleomorphism and polymegathism $(40 \times)$.

Abbreviations: OD, right eye; OS, left eye.

scrolls and corneal edema. All patients underwent penetrating keratoplasty and the immunohistochemistry and electron microscopic studies of the retrocorneal scrolls were performed. No studies described the findings from an anterior segment evaluation.

Histopathologic studies of the corneal buttons reported that the scrolls adhered to the posterior corneal surface, extended into the anterior chamber, ${ }^{2}$ and were reconnected to a thickened Descemet's membrane by stalks. ${ }^{5}$ The retrocorneal scrolls were comprised of a multilayered, amorphous, acellular matrix, ${ }^{5}$ lined by attenuated endothelium and macrophages with polymorphic pigment granules adhering to the endothelium in some areas. ${ }^{2}$ The endothelium was present, but it was thin and attenuated..$^{5}$ Cellular components

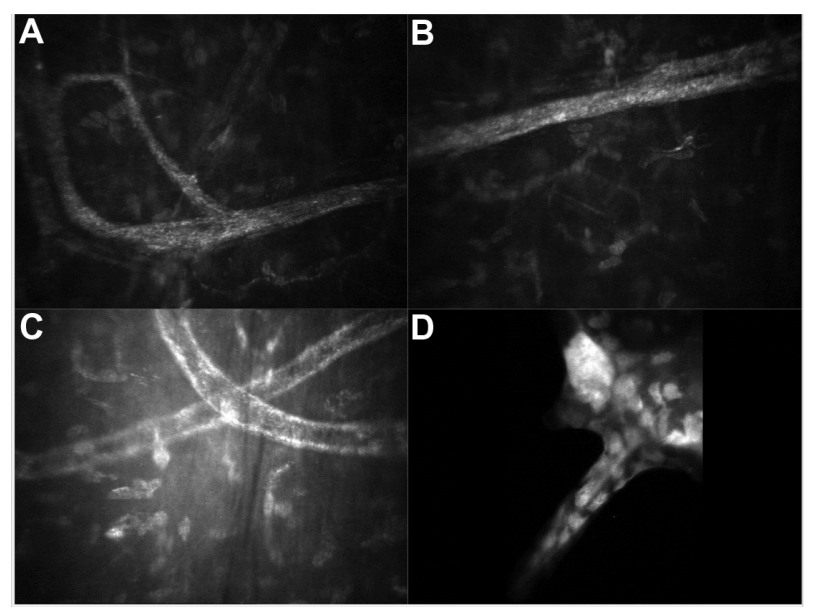

Figure 5 Confocal microscopy of the right eye shows a Descemet's scroll. Notes: The scrolls appeared as (A and $\mathbf{B})$ tubular structures with (C) outer hyperreflectivity and inner hyporeflectivity. (D) Some areas show cellular components on the surface. were observed only on the surface of the scrolls, but not between the extracellular matrix layers., ${ }^{3,5}$ Staining with Azan-Mallory, Alcian blue, and periodic acid-Schiff indicated that the scrolls contained collagenous components and glycosaminoglycans. ${ }^{3}$

Immunoelectron microscopy of the scrolls showed positive staining for collagens type I, III, IV, VI, VIII, fibronectin, laminin, and decorin, but not for $\alpha$-smooth muscle actin. ${ }^{5}$ The fibroblast-like corneal endothelial cells were positive for vimentin, collagen type I, fibronectin, and heat shock protein 47 , but not for $\alpha$-smooth muscle actin, proliferating cell nuclear antigen, or Ki67, indicating that they were not proliferating. ${ }^{3}$

Scanning electron microscopy showed abnormal endothelial cells with cytoplasmic projections and a central cytoplasmic elevation representing a presumed nucleus. The cells also exhibited a loss of intercellular interdigitation. ${ }^{3}$ Transmission electron microscopy with hematoxylin and eosin staining of a retrocorneal scroll showed a central irregular core with concentric layers of basement membranelike material. The centrum was an amorphous core with fine granular material, and there were multiple layers of extracellular matrix material between the core and the attenuated endothelium. ${ }^{5}$

In the current report, the patient had good VA and deferred the need for keratoplasty. Hence, we did not obtain specimens of the corneal tissue for histopathologic examination. The confocal microscopy showed pleomorphism and polymegathism with a low endothelial cell density, which suggested the risk for corneal decompensation after intraocular surgery. We did not observe guttae by confocal microscopy, despite observing it during the slit-lamp examination. Waring et $\mathrm{al}^{4}$ reported guttate in $88 \%$ of their case series. More areas may need to be examined. The central hollow area and the cellular component visualized by confocal microscopy might correspond to the amorphous core and the surrounding abnormal endothelial cells or to the macrophages in the histopathologic findings.

AS-OCT showed Descemet's membrane thickening and a tubular structure extending from Descemet's membrane into the anterior chamber. The border of the tubular structure appeared to be hyperreflective, while the inner portion appeared to be hyporeflective, as seen in confocal microscopy. However, no cellular component was observed. The UBM finding was similar to that of AS-OCT; however, the resolution and magnification of the UBM images were lower compared to those of the AS-OCT images. 


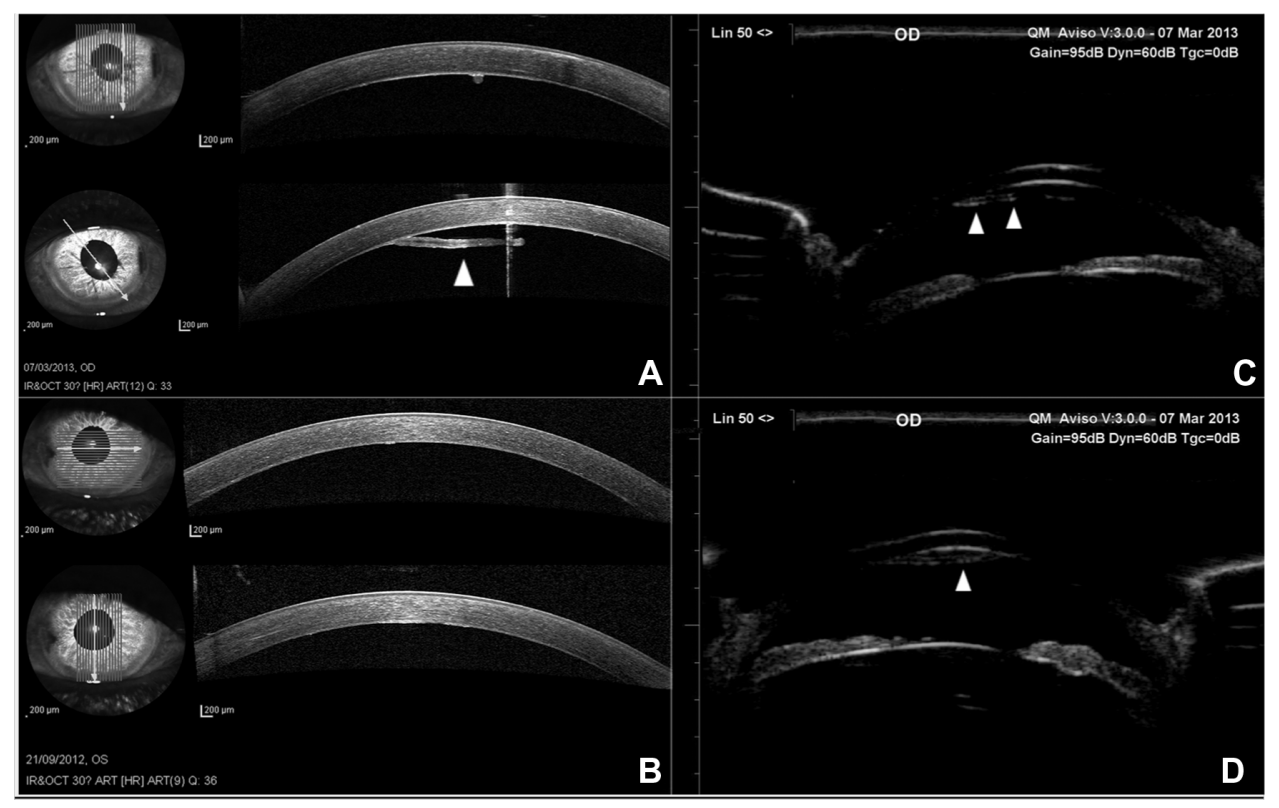

Figure 6 OD and OS AS-OCT and OD ultrasound biomicroscope.

Notes: $(\mathbf{A})$ OD and (B) OS AS-OCT show a thickened Descemet's membrane; (A) the scroll extended from the posterior corneal surface into the anterior chamber. Arrowhead shows the rod-shaped Descemet's scroll. (C and D) UBM of the right eye shows Descemet's scrolls. Arrowheads show the rod-shaped Descemet's scroll. Abbreviations: OD, right eye; OS, left eye; AS-OCT, anterior segment optical coherence tomography; UBM, ultrasound biomicroscopy.

\section{Conclusion}

In conclusion, we present a case of syphilitic interstitial keratitis with retrocorneal scrolls and its characteristic finding from the anterior segment examination. Without obtaining corneal tissue for evaluation, the noninvasive anterior segment study provided insight into the underlying histopathology and natural disease history. Since some reports have identified corneal decompensation after cataract surgery, early recognition of the compromised endothelium and abnormal structures is beneficial to the preoperative evaluation and prevention of complications.

\section{Acknowledgments}

We would like to thank Miss Sareedawan Nuchsiri and Miss Piyada Saehout for their technical assistance and performance of the confocal microscopy.

\section{Disclosure}

The authors report no conflicts of interest in this work.

\section{References}

1. Krachmer JH, Mannis MJ, Holland EJ. Cornea. 3rd ed. Philadelphia, PA: Mosby Elsevier; 2011.

2. Scattergood KD, Green WR, Hirst LW. Scrolls of Descemet's membrane in healed syphilitic interstitial keratitis. Ophthalmology. 1983;90(12): $1518-1523$.

3. Kawaguchi R, Saika S, Wakayama M, Ooshima A, Ohnishi Y, Yabe H. Extracellular matrix components in a case of retrocorneal membrane associated with syphilitic interstitial keratitis. Cornea. 2001;20(1):100-103.

4. Waring GO, Font RL, Rodrigues MM, Mulberger RD. Alterations of Descemet's membrane in interstitial keratitis. Am J Ophthalmol. 1976; 81(6):773-785.

5. Dogru M, Kato N, Matsumoto Y, et al. Immunohistochemistry and electron microscopy of retrocorneal scrolls in syphilitic interstitial keratitis. Curr Eye Res. 2007;32(10):863-870.

6. Alexander RA, Rahi AH. Scrolls of Descemet's membrane as unusual giant nodules on the posterior cornea: histochemical and ultrastructural findings. Br J Ophthalmol. 1986;70(2):126-131.

7. Honig MA, Barraquer J, Perry HD, Riquelme JL, Green WR. Forceps and vacuum injuries to the cornea: histopathologic features of twelve cases and review of the literature. Cornea. 1996;15(5):463-472.
International Medical Case Reports Journal

\section{Publish your work in this journal}

The International Medical Case Reports Journal is an international, peer-reviewed open-access journal publishing original case reports from all medical specialties. Previously unpublished medical posters are also accepted relating to any area of clinical or preclinical science. Submissions should not normally exceed 2,000 words or

\section{Dovepress}

4 published pages including figures, diagrams and references. The manuscript management system is completely online and includes a very quick and fair peer-review system, which is all easy to use. Visit http://www.dovepress.com/testimonials.php to read real quotes from published authors. 